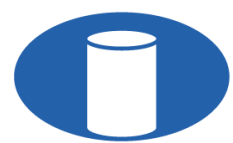

IBRACON

ORIGINAL ARTICLE

\title{
Reinforced concrete structures strengthened with CFRP (ACl $x$ FIB) - Recommendations for bending design criteria
}

\author{
Reforço de estruturas de concreto armado com CFRP (ACI x FIB) - Recomendações \\ para dimensionamento à flexão \\ Igor Del Gaudio Orlando ${ }^{\mathrm{a}}$ \\ Túlio Nogueira Bittencourt ${ }^{\mathrm{a}}$ (D) \\ Leila Cristina Meneghetti ${ }^{\mathrm{a}}$

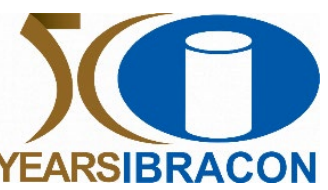

${ }^{a}$ Universidade de São Paulo - USP, Escola Politécnica, Departamento Engenharia de Estruturas e Geotécnica, São Paulo, SP, Brasil

Received 14 December 2020

Accepted 06 August 2021

\begin{abstract}
This work deals with the evaluation of the design criteria and security check (Ultimate Limit State - ULS) of the American (ACI-440.2R, 2017) and European (FIB Model Code, 2010) standards of reinforced concrete structures strengthened with Carbon Fiber Reinforced Polymers (CFRP), by the technique of Externally Bonded Reinforcement (EBR). It is intended to evaluate if, for a given database of 64 experimental tests of beams and slabs, the obtained results respect the safety conditions according to the mentioned standards, to increase the efficiency of this reinforcement technique and to lead to the establishment of regulatory design criteria in Brazil. Results show a conservative match among experimental and theoretical values calculated according to the two guidelines and it is concluded that a future regulation in Brazil on this subject should be based on the FIB Model Code.
\end{abstract}

Keywords: carbon fiber reinforced polymers (CFRP), bending strengthening, ACI-440 (2017), FIB Model Code (2010), regulation in Brazil.

\begin{abstract}
Resumo: Este trabalho trata da avaliação dos critérios de dimensionamento e verificação de segurança ao Estado Limite Último (ELU) da norma americana (ACI-440.2R, 2017) e europeia (FIB Model Code, 2010) de estruturas de concreto armado reforçadas à flexão com Polímeros Reforçados com Fibras de Carbono (CFRP), pela técnica de colagem externa (EBR). Considerando uma dada base de 64 dados de ensaios experimentais de vigas e lajes, avaliou-se se os resultados obtidos respeitam as condições de segurança segundo as normas referidas, com o propósito de aumentar a eficiência dessa técnica de reforço e conduzir ao estabelecimento de critérios regulamentares de dimensionamento no Brasil. Os resultados obtidos mostram uma proximidade conservadora entre valores experimentais e teóricos calculadas de acordo com as duas recomendações e conclui-se que uma futura regulamentação no Brasil sobre esse tema deve-se ter como premissa o modelo do FIB Model Code.
\end{abstract}

Palavras-chave: polímeros reforçados com fibras de carbono (CFRP), reforço à flexão, ACI-440 (2017), FIB Model Code (2010), regulamentação no Brasil.

How to cite: I. D. G. Orlando, T. N. Bittencourt, and L. C. Meneghetti, "Reinforced concrete structures strengthened with CFRP (ACI x FIB) Recommendations for bending design criteria," Rev. IBRACON Estrut. Mater., vol. 15, no. 2, e15204, 2022, https://doi.org/10.1590/S198341952022000200004

\section{INTRODUCTION}

In recent years, the development of new materials, the improvement of execution techniques, and the greater knowledge about the behavior of structures, in conjunction with a greater concern about the durability of constructions, have made repair, strengthening, and retrofit of concrete elements one of the most evolved areas in engineering. Structures are required to sustain critical loads under challenging environmental conditions such as heavy traffic 
density, impact from debris flow and highly corrosive environments. Therefore, strengthening is frequently required in reinforced concrete (RC) structures to meet the adequate strength requirements and extend the service life [1].

As described by Toutanji et al. [2], one of the techniques developed during the last decades to strength reinforced concrete beams in bending is the use of fiber-reinforced polymers (FRP) through the externally bonded reinforcement (EBR) technique. Increases in the strength of global flexion from 10 to $160 \%$ were reported in prior studies [3]-[8]. These materials feature good properties of non-corrosiveness; high longitudinal tensile strength, high stiffness, high strength-to-weight ratio, insect and fungal resistance chemical attack resistance, low thermal transmissibility, and simple installation; which supported their popularization in the structural reinforcement market [9]-[13].

Currently, the use of FRPs in buildings and bridge repair, strengthening and maintenance is most pronounced due to their efficient and economical nature [14], [15]. In recent years, the construction sector has become one of the world's largest consumers of polymer composites [16]-[20], indicating that FRP materials are part of the modern construction industry.

However, in Brazil there is still no specific standard for strengthening projects related to the use of composite materials. Strengthening concrete structures with FRP are carried out following international standards and recommendations, as well as manufacturer specifications [21]. The main design recommendations for strengthening with FRP are the American Concrete Institute (ACI) - Committee 440 [22] and the European standard "FIB Model Code - Task Group 9.3" [23].

To resolve this issue and propose a Brazilian guideline, it is necessary to evaluate and discuss the design parameters and methodologies suggested in the literature and compare them with a variety of experimental results.

This study aims to provide fundamentals to assist in deciding about the design procedures to be adopted in Brazil in structural strengthening projects with FRP composites. Therefore, discussion and evaluation of both design criteria and safety factor assessment (Ultimate Limit State - ULS) of ACI-440 [22] and FIB Model Code [23] are presented and some parameters and methodology highlighted as important to be considered in the future development of code design criteria in Brazil.

\section{STATE OF THE ART}

This section discusses analytical design models applied to projects of concrete beams bending strengthening with FRP.

\subsection{Basic design assumptions}

According to ACI 440 [22] and FIB [23], the following assumptions are applied in the strengthening of bending design elements:

- The strain in the concrete and reinforcement are directly proportional to their respective distances to the neutral axis of the section. Flat sections before loading remain flat after loading (Euler-Bernoulli's assumption);

- The maximum compression strain in the concrete is 0.003 (ACI) or 0.0035 (FIB);

- The tensile strength of concrete is neglected;

- The stress vs. strain diagram of the steel is elastic-linear until its yield point, followed by perfectly plastic behavior;

- It is admitted that FRP are characterized by an elastic-linear stress-strain behavior to rupture;

- The shear strain in the adhesive layer is neglected, given that this layer is very thin with small variation in its thickness.

\subsection{Rupture modes of FRP systems}

The bending capacity of a reinforced member is related to its rupture mode. The following rupture modes may occur in a bending member strengthened with FRP:

- Crushing of the compressed concrete (CC) before the yielding of reinforcement;

- Yielding of reinforcement (FY) followed by the failure of the FRP system (FR);

- Yielding of reinforcement (FY) followed by the concrete crushing (CC);

- Debonding of the FRP (FD);

- Delamination of the FRP system from concrete substrate (FD).

According to Juvandes [24], the first three types constitute the group of modes where the section presents a perfect bond between adhesive, FRP and concrete interfaces until rupture (classic rupture - RC). The two remaining cases (FD) 
define the group of premature failure of the FRP (premature rupture - RP). Debonding is known to occur at low axial strain level of FRP, thus externally bonded systems often do not yield full tensile strength of FRP [25]-[27].

As shown by Kalfat et al. [28], different types of intermediate anchorages, including U-jacket anchors, mechanically fastened anchors, and FRP anchors, have been used to prevent early debonding failure. Metallic clamps have been used to prevent delamination and to increase ductility [29]. These anchors delay debonding by enabling the continuation of the load path between FRP concrete and increasing bond strength [30].

\subsection{ACI-440.2R-17 model [22]}

The strength design approach requires that the design flexural strength of a member exceed its required factored moment, as indicated by Equation 1:

$\varnothing M_{n} \geq M_{u}$

where $\varnothing=$ strength reduction factor; $M_{n}=$ nominal flexural strength; and $M_{u}=$ factored moment at a section.

As described previously, one of the consequences of the use of FRP in strengthened concrete structures is the reduction of the ductility of the original element. In some cases, this loss is negligible, but sections that may have significant ductility losses should be checked. For reinforced concrete elements, adequate ductility is achieved if the strain in the steel is at least equal to 0.005 at the instant of the concrete rupture or the debonding/delamination of the FRP system,

ACI-440 [22] recommends using the strength reduction factor $(\varnothing)$, which is a function of the yielding strain of the steel $\left(\varepsilon_{s y}\right)$ and the unitary net tensile strain in steel $\left(\varepsilon_{t}\right)$ according to Equation 2, 3 and 4:

$\varnothing=0.90$ para $\varepsilon_{t} \geq 0.005$

$\varnothing=0.65+\frac{0.25\left(\varepsilon_{t}-\varepsilon_{s y}\right)}{0.005-\varepsilon_{s y}} \quad$ to $\quad \varepsilon_{s y}<\varepsilon_{t}<0.005$

$\varnothing=0.65$ to $\varepsilon_{t} \leq 0.005 \varepsilon_{s y}$

The bending strength capacity of the section of an element strengthened with FRP can be determined through the compatibility of strain, balance of internal forces and control of the failure mode. The nominal flexural strength capacity of the section $\left(M_{n}\right)$ can be calculated as Equation 5:

$M_{n}=A_{s} f_{S}\left(d-\frac{\beta_{1} c}{2}\right)+\psi_{f} A_{f} f_{f e}\left(h-\frac{\beta_{1} c}{2}\right)+A^{\prime}{ }_{s} f^{\prime}{ }_{S}\left(\frac{\beta_{1} c}{2}-d^{\prime}\right)$

where $A_{S}=$ area of nonprestressed steel reinforcement; $A_{s}^{\prime}=$ area of the compression reinforcement of the section; $f_{s}=$ stress in steel reinforcement; $h=$ total height of the section; $d=$ useful height of section; $c=$ position of the neutral axis; $A_{f}=$ FRP area; and $f_{f e}=$ effective stress in FRP.

The application of the coefficient of reduction in the strength of the FRP, denoted by $\psi_{f}$, in the portion that simulates the contribution of FRP to the resistant moment is defined in ACI 440 [22], item "10.2.10". According to Okeil et al. [31] it is based on reliability analyses that in turn is based on the statistically calibrated properties of the bending strength.

The terms $\alpha_{1}$ and $\beta_{1}$ in the equations below are parameters that define a rectangular stress block in concrete equivalent to a non-linear stress distribution. Considering $\alpha_{I}=0.85$ (Whitney stress block) it is possible to obtain reasonably accurate results for a rectangular section. In addition, $\beta_{1}=0.85$, when $17 \mathrm{MPa}<f^{\prime}{ }_{c}<28 \mathrm{MPa}$. For $f_{c}^{\prime}>28 \mathrm{MPa}$, the value of $\beta_{l}$ is provided by the general expression (ACI-318), presented in Equation 6: 
$\beta_{1}=1.05-0.05\left(\frac{f^{\prime}{ }_{c}}{7}\right)$

Where $\beta_{1}>0,65$. The depth of the neutral axis (c) is found by satisfying the internal balance of forces and the compatibility of strain according to the following Equation 7:

$c=\left(\frac{A_{s} f_{s}+A_{f} f_{e}-A^{\prime}{ }_{s} f^{\prime}{ }_{s}}{\alpha_{1} f^{\prime}{ }_{c} \beta b}\right)$

The effective strain $\left(\varepsilon_{\mathrm{fe}}\right)$ that can be achieved by the FRP is defined as Equation 8 :

$\varepsilon_{f e}=\varepsilon_{c u}\left(\frac{d_{f}-c}{c}\right)-\varepsilon_{b i} \leq \varepsilon_{f d}$

Where $\varepsilon_{b i}=$ pre-existing strain when the FRP is installed; $d_{f}=$ effective depth of FRP; $\varepsilon_{c u}=$ ultimate axial strain of the unconfined concrete corresponding to $0.85 f^{\prime}{ }_{c 0}$; and $\varepsilon_{f e}=$ effective strain in FRP reinforcement attained at failure.

Regardless of where the neutral axis of the analyzed section is, the failure controlled by the FRP debonding may govern the procedures. Thus, to prevent crack induced debonding failure mode, the effective strain in FRP should be limited to the strain at which debonding may occur as defined in Equation 9:

$\varepsilon_{f d}=0.412 \sqrt{\frac{f_{c}^{\prime}}{n E_{f} t_{f}}} \leq 0.9 \varepsilon_{f u}$

where the maximum allowable strain in FRP $\left(\varepsilon_{f d}\right)$ is a function of the thickness of each layer of FRP $\left(t_{f}\right)$, the number of layers of FRP $(n)$ and the modulus of elasticity of FRP $\left(E_{f}\right)$.

As reported by Arduini and Nanni [32], it's important to note that carbon fiber stiffness, fiber direction, and number of plies can significantly affect the performance of strengthened beams. Toutanji et al. [33] observed that not only ductility of strengthened beams tends to significantly reduce with the increase on the number of bonded sheets, but also the failure mode of strengthened-specimens changes. For instance, wrapping of FRP sheets perpendicular to edges of strengthened beam (and on top of longitudinally applied FRP sheets) can be effective in anchoring bonded CFRP sheets and delays debonding of FRP strengthening system. However, though anchor system enhanced the capacity of the strengthened beam significantly, it reduces the overall ductility [34].

\subsection{FIB Model Code (2010) [23]}

In the FIB Model Code 2010 [23], the calculation of the resistance design moment $\left(M_{R d}\right)$ of the strengthened section is also based on the design principles of reinforced concrete, according to the Equation 10:

$M_{R d}=A_{S 1} f_{y d}(d-\lambda x)+A_{f} E_{f} \varepsilon_{f}(h-\lambda x)+A_{S 2} E_{S} \varepsilon_{S 2}\left(\lambda x-d_{2}\right)$

The neutral axis is determined from the compatibility of the strains and internal equilibrium of forces as Equation 11:

$\eta f_{c d} b x+A_{s 2} E_{s} \varepsilon_{s 2}=A_{s 1} f_{y d}+A_{f} E_{f u} \varepsilon_{f}$ 
where the terms $\lambda$ e $\eta$ in Equations 10 and 11 are parameters that define a rectangular stress block in the concrete equivalent to a non-linear stress distribution, according to Equations 12 and 13 below. These equations provide reasonably accurate results for a rectangular section.

$$
\begin{aligned}
& \lambda= \begin{cases}0.8 & \text { to } f_{c k} \leq 50 \mathrm{MPa} \\
0.8-\frac{f_{c k}-50}{400} & \text { to } 50 \leq f_{c k} \leq 100 \mathrm{MPa}\end{cases} \\
& \eta= \begin{cases}1.0 & \text { to } f_{c k} \leq 50 \mathrm{MPa} \\
1.0-\frac{f_{c k}-50}{200} & \text { to } 50 \leq f_{c k} \leq 100 \mathrm{MPa}\end{cases}
\end{aligned}
$$

As sudden failures with little or no warning are undesirable, the ductility index of the strengthened element must exceed a certain value. With respect to this issue, the FIB Model Code [23] gives the following limitation on the depth of the compression zone at ultimate: $\xi \leq 0,45$ (for concrete type C50/60 or lower) or $\xi \leq 0,35$ for concrete above C55/67, where:

$$
\xi=\frac{c}{d}
$$

The maximum allowable strain in the FRP to prevent debounding failure due to concrete crack may not exceed the limit indicated by the FIB Model Code [23] and supplemented by the FIB-14 [35] through the Equations 15 and 16:

$$
\varepsilon_{f d} \leq 0.9 \varepsilon_{f u}
$$

$$
f_{f d}=\frac{f_{f k}}{y_{f}}
$$

Where $y_{f}$ (FRP material partial safety factor) is given in Table 3.1 of FIB-14 [35], with $y_{f}=1.35$ for any strengthening system with low-quality control of the application on the construction site.

The maximum tensile stress in the FRP limited by the connection to concrete in a single anchorage zone (not cracked) is given by Equations 17 and 18 of FIB Model Code, 2010 [23]:

$$
\begin{aligned}
& f_{f b m}=K_{m} K_{b} \beta_{L} \sqrt[2]{\frac{2 E_{f}}{t_{f}} \cdot f_{c m}{ }^{2 / 3}} \\
& k_{b}=\sqrt[2]{\frac{2-b_{f} / b}{1+b_{f} / b}} \geq 1
\end{aligned}
$$

where $t_{f}=$ thickness of the FRP layer FRP; $\beta_{L}=$ is the anchorage length factor of the FRP; $K_{b}$ : structure shape factor; $b_{f}$ : FRP section width; $b$ : width of the concrete section; and $E_{f}$ : the modulus of elasticity of the FRP.

\section{DESCRIPTION OF DATA EXTRACTED FROM THE LITERATURE}

For further investigation of the design parameters, a set of 64 experimental data were selected according to the following criteria:

- Type of structural element: slab and beam; 
- Reinforcement technique: EBR;

- Type of reinforcement: carbon fiber reinforced polymers (CFRP) (sheet and laminates).

The data, which are shown in Tables 1 and 2, were collected from the following experimental studies:

- Slabs: Juvandes [24], Dias [37].

- Beams: Gamino et al. [21], Dias [37], Beber [38], Brosens [39], Matthys [40], Pinto [41], Travassos [42].

Note that CFRP was employed because this type of reinforcement is widely used and studied, resulting in a greater sampling of available experimental elements. Tables 1 and 2 include the failure modes observed for the 64 collected data and the level of strain of the CFRP at the moment of rupture $\left(\varepsilon_{f u}\right)$. For 12 experimental beam data, these strain values are unknown. It can be observed that in both slabs and beams, approximately $40 \%$ of the samples presented classic ruptures.

Table 1. Summary of the 12 experimental slab data used in this study*.

Reinforced concrete element

CFRP System

\begin{tabular}{|c|c|c|c|c|c|c|c|c|c|c|c|c|c|c|c|c|c|}
\hline \multirow{3}{*}{ Author } & \multirow{3}{*}{$\begin{array}{l}\text { Exp. } \\
\text { Data }\end{array}$} & \multirow{3}{*}{ Type } & \multirow{3}{*}{$\frac{\bar{b}}{(\mathrm{~cm})}$} & \multirow{3}{*}{$\frac{\mathrm{h}}{(\mathrm{cm})}$} & \multirow{3}{*}{$\frac{A_{s}}{\left(\mathrm{~cm}^{2}\right)}$} & \multirow{3}{*}{$\frac{\rho_{\mathrm{s}}}{(\%)}$} & \multirow{3}{*}{$\frac{\mathrm{f}^{\mathrm{cil}} \mathrm{cm}}{(\mathrm{MPa})}$} & \multirow{3}{*}{$\frac{E_{f 1}}{(G P a)}$} & \multirow{3}{*}{$\frac{\varepsilon_{\mathrm{fk} \mathbf{1}}}{(\% \mathrm{o})}$} & \multirow{3}{*}{$\frac{b_{\mathbf{f} 1}}{(\mathrm{~mm})}$} & \multirow{3}{*}{$\frac{t_{f 1}}{(\mathbf{m m})}$} & \multirow{3}{*}{$\mathbf{n}_{\text {bf1 }}$} & \multirow{3}{*}{$\mathbf{n}_{\mathbf{f f} \mathbf{1}}$} & \multirow{3}{*}{$\frac{A_{f 1}}{\left(\mathrm{~cm}^{2}\right)}$} & \multirow{3}{*}{$\frac{\rho_{f}}{(\%)}$} & \multirow{3}{*}{$\frac{\varepsilon_{f u}}{(\%)}$} & \multirow{3}{*}{$\frac{\text { Type }}{\text { Rupt. }}$} \\
\hline & & & & & & & & & & & & & & & & & \\
\hline & & & & & & & & & & & & & & & & & \\
\hline \multirow{8}{*}{$\begin{array}{l}\frac{\pi}{n} \\
\frac{\pi}{0}\end{array}$} & LA3R & S. & 44.0 & 7.6 & 0.85 & $0.31 \%$ & 63.3 & 230 & 15 & 140 & 0.11 & 1 & 2 & 0.311 & $0.09 \%$ & 11.1 & $\mathrm{RC}$ \\
\hline & LB1R & S. & 44.0 & 8.1 & 0.85 & $0.28 \%$ & 63.3 & 230 & 15 & 140 & 0.11 & 1 & 2 & 0.311 & $0.09 \%$ & 12.0 & $\mathrm{RC}$ \\
\hline & LA4S & L. & 44.0 & 8.0 & 0.85 & $0.29 \%$ & 63.3 & 160 & 20 & 32 & 1.20 & 1 & 1 & 0.384 & $0.11 \%$ & 9.7 & $\mathrm{RP}$ \\
\hline & LB2S & L. & 44.0 & 8.5 & 0.85 & $0.27 \%$ & 63.3 & 160 & 20 & 32 & 1.20 & 1 & 1 & 0.384 & $0.10 \%$ & 9.2 & $\mathrm{RP}$ \\
\hline & LD3BL & L. & 44.0 & 8.5 & 0.85 & $0.27 \%$ & 49.7 & 150 & 14 & 32 & 1.40 & 1 & 1 & 0.448 & $0.12 \%$ & 9.6 & $\mathrm{RP}$ \\
\hline & LD4BL & L. & 44.0 & 8.1 & 0.85 & $0.28 \%$ & 49.7 & 150 & 14 & 32 & 1.40 & 1 & 1 & 0.448 & $0.13 \%$ & 10.4 & $\mathrm{RP}$ \\
\hline & LE3I & L. & 44.0 & 8.2 & 0.85 & $0.28 \%$ & 49.7 & 160 & 15 & 32 & 1.40 & 1 & 1 & 0.448 & $0.12 \%$ & 8.6 & $\mathrm{RP}$ \\
\hline & LE4I & L. & 44.0 & 7.8 & 0.85 & $0.30 \%$ & 49.7 & 160 & 15 & 32 & 1.40 & 1 & 1 & 0.448 & $0.13 \%$ & 10.2 & $\mathrm{RP}$ \\
\hline \multirow{4}{*}{ 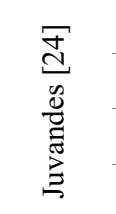 } & LC3R & S. & 44.0 & 8.1 & 0.85 & $0.28 \%$ & 63.3 & 230 & 15 & 140 & 0.11 & 1 & 2 & 0.311 & $0.09 \%$ & 10.9 & $\mathrm{RC}$ \\
\hline & LC4R & S. & 44.0 & 7.7 & 0.85 & $0.30 \%$ & 63.3 & 230 & 15 & 140 & 0.11 & 1 & 2 & 0.311 & $0.09 \%$ & 10.3 & $\mathrm{RC}$ \\
\hline & LC1S & $\mathrm{L}$. & 44.0 & 8.1 & 0.85 & $0.28 \%$ & 63.3 & 160 & 20 & 32 & 1.20 & 1 & 1 & 0.384 & $0.11 \%$ & 10.3 & $\mathrm{RP}$ \\
\hline & LC2S & $\mathrm{L}$. & 44.0 & 8.4 & 0.85 & $0.27 \%$ & 63.3 & 160 & 20 & 32 & 1.20 & 1 & 1 & 0.384 & $0.10 \%$ & 11.8 & $\mathrm{RP}$ \\
\hline
\end{tabular}

$* \mathrm{RC}=$ classical failure; $\mathrm{RP}=$ premature failure; $\varepsilon_{\mathrm{fu}}=$ ultimate FRP strain during tests; $\rho_{\mathrm{f}}=$ FRP reinforcement ratio; $\rho_{\mathrm{s}}=$ reinforcement ratio; feil ${ }_{\mathrm{cm}}=$ mean compressive strength of concrete cylinders; $\mathrm{L}=$ Laminate; $\mathrm{S}=$ Sheet

Table 2. Summary of the 52 experimental beams data used in this study.

\begin{tabular}{|c|c|c|c|c|c|c|c|c|c|c|c|c|c|c|c|c|c|}
\hline \multirow{3}{*}{ Author } & \multirow{3}{*}{$\begin{array}{l}\text { Exp. } \\
\text { Data }\end{array}$} & \multirow{3}{*}{ Type } & \multicolumn{5}{|c|}{ Reinforced concrete element } & \multicolumn{10}{|c|}{ CFRP System } \\
\hline & & & b & $\mathbf{h}$ & As & $\rho_{s}$ & $\mathbf{f}^{\mathrm{cil}}{ }_{\mathrm{cm}}$ & $\mathbf{E}_{\boldsymbol{f} \mathbf{1}}$ & $\varepsilon_{\mathrm{fk} 1}$ & $\mathbf{b}_{\mathbf{f} 1}$ & $\mathbf{t}_{\mathbf{f} 1}$ & & & $\mathbf{A}_{\mathrm{f} 1}$ & $\rho_{\mathrm{f}}$ & $\varepsilon_{f u}$ & Type \\
\hline & & & (cm) & (cm) & $\left(\mathrm{cm}^{2}\right)$ & $(\%)$ & (MPa) & (GPa) & (\%) & $(\mathbf{m m})$ & $(\mathbf{m m})$ & & & $\left(\mathrm{cm}^{2}\right)$ & $(\%)$ & $(\%)$ & Rupt. \\
\hline \multirow{4}{*}{ 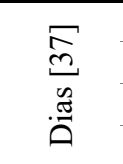 } & V2 & $\mathrm{S}$ & 12.0 & 18.0 & 1.01 & $0.52 \%$ & 41.0 & 240 & 15 & 70 & 0.11 & 1 & 2 & 0.155 & $0.07 \%$ & 8.1 & $\mathrm{RP}$ \\
\hline & V3 & $\mathrm{S}$ & 12.0 & 18.0 & 1.01 & $52 \%$ & & 24 & & & 0. & & & 0.155 & $0.07 \%$ & 8.1 & $\mathrm{C}$ \\
\hline & V4 & $\mathrm{L}$ & 12.0 & 18.0 & 1.01 & $52 \%$ & 41 & 200 & 1 & 20 & 1.40 & & 1 & 0.280 & $0.13 \%$ & 6.9 & RP \\
\hline & V6 & $\mathrm{L}$ & 12.0 & 18.0 & 1.01 & $52 \%$ & 41.0 & 200 & 11 & 20 & 1.40 & 1 & 1 & 0.280 & $0.13 \%$ & 7.2 & $\mathrm{RP}$ \\
\hline \multirow{3}{*}{ 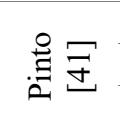 } & V1 & $\mathrm{L}$ & 15.0 & 45.0 & 6.03 & $3 \%$ & & 16 & 17 & 50 & 1.2 & 2 & 1 & 1.200 & $0.18 \%$ & 5.2 & $\mathrm{RP}$ \\
\hline & V3 & $\mathrm{L}$ & 15.0 & 45.0 & 6.03 & $0.98 \%$ & 38.3 & 165 & 17 & 50 & 1.20 & 3 & 1 & 1.800 & $0.27 \%$ & 5.4 & $\mathrm{RP}$ \\
\hline & V5 & $\mathrm{L}$ & 15.0 & 45.0 & 6.03 & $0.97 \%$ & 34.7 & 165 & 17 & 50 & 1.20 & 3 & 1 & 1.800 & $0.44 \%$ & 4.6 & $\mathrm{RC}$ \\
\hline \multirow{4}{*}{ 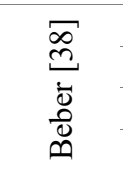 } & VR3 & $\mathrm{L}$ & 12.0 & 25.0 & 1.57 & $0.57 \%$ & 33.6 & 230 & 15 & 100 & 0.11 & 1 & 1 & 0.111 & $0.04 \%$ & 5.6 & $\mathrm{RC}$ \\
\hline & VR4 & $\mathrm{L}$ & 12.0 & 25.0 & 1.57 & $0.57 \%$ & 33.6 & 230 & 15 & 100 & 0.11 & 1 & 1 & 0.111 & $0.04 \%$ & 7.1 & $\mathrm{RC}$ \\
\hline & VR5 & $\mathrm{L}$ & 12.0 & 25.0 & 1.57 & $0.57 \%$ & 33.6 & 230 & 15 & 100 & 0.11 & 1 & 4 & 0.444 & $0.15 \%$ & 7.1 & $\mathrm{RP}$ \\
\hline & VR6 & $\mathrm{L}$ & 12.0 & 25.0 & 1.57 & $0.57 \%$ & 33.6 & 230 & 15 & 100 & 0.11 & 1 & 4 & 0.444 & $0.15 \%$ & 7.5 & $\mathrm{RP}$ \\
\hline
\end{tabular}


Table 2. Continued...

Reinforced concrete element

\section{CFRP System}

\begin{tabular}{|c|c|c|c|c|c|c|c|c|c|c|c|c|c|c|c|c|c|}
\hline \multirow[t]{2}{*}{ Author } & \multirow[t]{2}{*}{$\begin{array}{l}\text { Exp. } \\
\text { Data }\end{array}$} & \multirow[t]{2}{*}{ Type } & b & h & As & $\rho_{\mathrm{s}}$ & $\mathbf{f}^{\mathrm{cil}}{ }_{\mathrm{cm}}$ & \multicolumn{2}{|c|}{$-E_{\mathbf{f} 1} \varepsilon_{\mathbf{f k} 1}$} & \multirow{2}{*}{\multicolumn{2}{|c|}{$\frac{b_{f 1}}{(m m)} \frac{t_{f 1}}{(m m)}$}} & \multirow{2}{*}{$\mathbf{n}_{\text {bf1 }}$} & \multirow{2}{*}{$\mathbf{n}_{\text {If1 }}$} & \multirow{2}{*}{$\frac{\mathrm{A}_{\mathrm{f} 1}}{\left(\mathrm{~cm}^{2}\right)}$} & \multirow{2}{*}{$\frac{\rho f}{(\%)}$} & \multirow{2}{*}{$\frac{\varepsilon_{\mathrm{fu}}}{(\% \mathrm{o})}$} & \multirow{2}{*}{$\frac{\text { Type }}{\text { Rupt. }}$} \\
\hline & & & (cm) & (cm) & $\left(\mathrm{cm}^{2}\right)$ & $(\%)$ & (MPa) & (GPa) & (\%o) & & & & & & & & \\
\hline & VR7 & L & 12.0 & 25.0 & 1.57 & $0.57 \%$ & 33.6 & 230 & 15 & 100 & 0.11 & 1 & 7 & 0.777 & $0.26 \%$ & 5.2 & RP \\
\hline & VR8 & $\mathrm{L}$ & 12.0 & 25.0 & 1.57 & $0.57 \%$ & 33.6 & 230 & 15 & 100 & 0.11 & 1 & 7 & 0.777 & $0.26 \%$ & 5.6 & $\mathrm{RP}$ \\
\hline & VR9 & $\mathrm{L}$ & 12.0 & 25.0 & 1.57 & $0.57 \%$ & 33.6 & 230 & 15 & 100 & 0.11 & 1 & 10 & 1.110 & $0.37 \%$ & 4.8 & RP \\
\hline & VR10 & $\mathrm{L}$ & 12.0 & 25.0 & 1.57 & $0.57 \%$ & 33.6 & 230 & 15 & 100 & 0.11 & 1 & 10 & 1.110 & $0.37 \%$ & 4.7 & $\mathrm{RP}$ \\
\hline \multirow{8}{*}{ 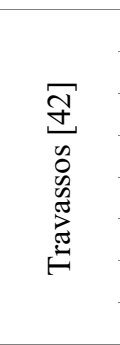 } & A12 & $\mathrm{L}$ & 20.0 & 40.0 & 4.02 & $0.59 \%$ & 38.5 & & 16 & 200 & 0.11 & 1 & 3 & 0.666 & $0.08 \%$ & 8.0 & $\mathrm{RP}$ \\
\hline & A14 & $\mathrm{L}$ & 20.0 & 40.0 & 4.02 & $0.59 \%$ & 38.5 & 242 & 16 & 200 & 0.11 & 1 & 1 & 0.222 & $0.03 \%$ & 6.2 & $\mathrm{RC}$ \\
\hline & A32 & L & 20.0 & 40.0 & 4.02 & $0.59 \%$ & 38.5 & 242 & 16 & 200 & 0.11 & 1 & 3 & 0.666 & $0.08 \%$ & 7.2 & $\mathrm{RP}$ \\
\hline & A33 & $\mathrm{L}$ & 20.0 & 40.0 & 4.02 & $0.59 \%$ & & & 16 & 200 & & 1 & 1 & & $0.03 \%$ & 3.2 & $\mathrm{RC}$ \\
\hline & A11 & $\mathrm{L}$ & & & & $0.59 \%$ & & & 16 & & & 1 & 1 & & & & $\mathrm{RC}$ \\
\hline & A21 & $\mathrm{L}$ & 20.0 & 40.0 & 9.42 & $1.39 \%$ & 34.4 & & 16 & 200 & & 1 & 1 & & $0.03 \%$ & 9.2 & $\mathrm{RC}$ \\
\hline & A31 & $\mathrm{L}$ & 20.0 & 40.0 & 4.02 & $0.59 \%$ & 34.4 & & 16 & 200 & 0.11 & 1 & 3 & 666 & $0.08 \%$ & 10.6 & $\mathrm{RP}$ \\
\hline & A34 & $\mathrm{L}$ & 20.0 & 0.0 & & $0.59 \%$ & 34. & & 16 & 20 & & 1 & 1 & 22 & $0.03 \%$ & 8.4 & $\mathrm{RC}$ \\
\hline \multirow{7}{*}{ 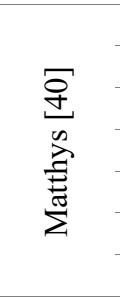 } & BF2 & $\mathrm{L}$ & 20.0 & 5.0 & & $0.96 \%$ & 36.5 & & 19 & 10 & & 1 & 1 & & $\%$ & 6.7 & $\mathrm{RP}$ \\
\hline & BF3 & $\mathrm{L}$ & 20.0 & 45.0 & 8.04 & $0.96 \%$ & 34.9 & 159 & 19 & 100 & 1.20 & 1 & 1 & 1.200 & $0.13 \%$ & 7.2 & $\mathrm{RP}$ \\
\hline & BF4 & $\mathrm{L}$ & 20.0 & 45.0 & 8.04 & $0.96 \%$ & 30.8 & 159 & 19 & 100 & 1.20 & 1 & 1 & 1.200 & $0.13 \%$ & 6.8 & $\mathrm{RP}$ \\
\hline & BF5 & $\mathrm{L}$ & 20.0 & 45.0 & 8.04 & $0.96 \%$ & 37.4 & 159 & 19 & 100 & 1.20 & 1 & 1 & 1.200 & $0.13 \%$ & 5.7 & $\mathrm{RP}$ \\
\hline & BF6 & $\mathrm{L}$ & 20.0 & 45.0 & 8.04 & $0.96 \%$ & 35.9 & 159 & 19 & 100 & 1.20 & 1 & 1 & 1.200 & $0.13 \%$ & 7.1 & RP \\
\hline & BF8 & $\mathrm{L}$ & 20.0 & 45.0 & 4.02 & $0.48 \%$ & 39.4 & 159 & 19 & 100 & 1.20 & 1 & 1 & 1.200 & $0.13 \%$ & 5.8 & $\mathrm{RC}$ \\
\hline & BF9 & $\mathrm{S}$ & 20.0 & 45.0 & 4.02 & $0.48 \%$ & 33.7 & 233 & 13 & 100 & 0.11 & 1 & 2 & 0.222 & $0.02 \%$ & 10.0 & $\mathrm{RP}$ \\
\hline \multirow{8}{*}{ 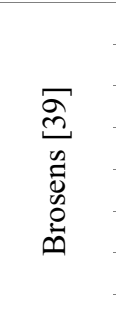 } & A1 & S & 12.5 & 22.5 & 1.01 & $0.41 \%$ & 41.0 & 2. & 15 & 75 & 0.1 & 1 & 2 & 51 & $0.09 \%$ & - & $\mathrm{RP}$ \\
\hline & B1 & S & 12.5 & 22.5 & 1.51 & $0.62 \%$ & 46.0 & 2. & 15 & 7 & 0.1 & 1 & 2 & 251 & $0.09 \%$ & & $\mathrm{RP}$ \\
\hline & $\mathrm{C} 1$ & S & 12.5 & 22.5 & 1.51 & $0.62 \%$ & 43.0 & 2. & 15 & 75 & 0.1 & 1 & 2 & 251 & $0.09 \%$ & - & $\mathrm{RP}$ \\
\hline & $\mathrm{C} 2$ & $\mathrm{~S}$ & & & & & & & & 7 & & 1 & 2 & & & & $\mathrm{RP}$ \\
\hline & D1 & $\mathrm{S}$ & & 22 & & 0.62 & & & & 75 & & 1 & 2 & & $\%$ & - & $\mathrm{RP}$ \\
\hline & E1 & $\mathrm{S}$ & 12.5 & 22.5 & 1.51 & 0.62 & 33.0 & 2. & 15 & 7 & 0.17 & 1 & 2 & 0.251 & $0.09 \%$ & - & $\mathrm{RP}$ \\
\hline & F1 & $\mathrm{S}$ & 12.5 & 22.5 & 1.29 & & & & 15 & 75 & 0.1 & 1 & 2 & 0.251 & $\%$ & - & $\mathrm{RP}$ \\
\hline & G1 & $\mathrm{S}$ & 12.5 & 22.5 & .07 & 0.85 & 43.0 & & 15 & 75 & 0.1 & 1 & 2 & 0.251 & $0.09 \%$ & - & $\mathrm{RP}$ \\
\hline \multirow{14}{*}{ 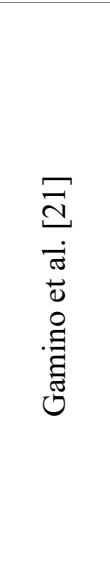 } & VR01 & $\mathrm{L}$ & 7.5 & 15.0 & 0.62 & $0.62 \%$ & 45.0 & 230 & 15 & 75 & 0.13 & 1 & 1 & 0.098 & $0.09 \%$ & 13.3 & $\mathrm{RC}$ \\
\hline & VR02 & $\mathrm{L}$ & 7.5 & 15.0 & 0.62 & $0.62 \%$ & 45.0 & 230 & 15 & 75 & 0.13 & 1 & 1 & 0.098 & $0.09 \%$ & - & $\mathrm{RC}$ \\
\hline & VR03 & $\mathrm{L}$ & 7.5 & 15.0 & 0.62 & $0.62 \%$ & 45.0 & 230 & 15 & 75 & 0.13 & 1 & 1 & 0.098 & $0.09 \%$ & 11.8 & $\mathrm{RC}$ \\
\hline & VR04 & $\mathrm{L}$ & 7.5 & 15.0 & 0.62 & $0.62 \%$ & 45.0 & 230 & 15 & 75 & 0.13 & 1 & 1 & 0.098 & $0.09 \%$ & 12.6 & $\mathrm{RC}$ \\
\hline & VR05 & $\mathrm{L}$ & 7.5 & 15.0 & 0.62 & $0.62 \%$ & 45.0 & 230 & 15 & 75 & 0.13 & 1 & 1 & 0.098 & $0.09 \%$ & 11.4 & $\mathrm{RC}$ \\
\hline & VR06 & $\mathrm{L}$ & 7.5 & 15.0 & 0.62 & $0.62 \%$ & 45.0 & 230 & 15 & 75 & & 1 & 1 & 0.098 & $0.09 \%$ & - & $\mathrm{RP}$ \\
\hline & VR07 & $\mathrm{L}$ & 7.5 & 15.0 & 0.62 & $0.62 \%$ & 45.0 & 230 & 15 & 75 & & 1 & 1 & 0.098 & $0.09 \%$ & - & $\mathrm{RP}$ \\
\hline & VR08 & $\mathrm{L}$ & 7.5 & 15.0 & 0.6 & $0.62 \%$ & 45 & 23 & 15 & 75 & & 1 & 1 & 0.098 & $0.09 \%$ & - & $\mathrm{RP}$ \\
\hline & VR09 & $\mathrm{S}$ & 7.5 & 15.0 & 0.62 & & & & 15 & 75 & & 1 & 1 & & $0.07 \%$ & 5.3 & $\mathrm{RC}$ \\
\hline & VR10 & $\mathrm{S}$ & 7.5 & 15.0 & 0.62 & & & & & 75 & & 1 & 1 & 0.083 & $.07 \%$ & 6.4 & $\mathrm{RC}$ \\
\hline & VR11 & S & 1.0 & 15.0 & 0.62 & $0.62 \%$ & 45.0 & 235 & 15 & 75 & 0.11 & 1 & 2 & 0.165 & $0.15 \%$ & 6.6 & $\mathrm{RC}$ \\
\hline & VR12 & $\mathrm{S}$ & 7.5 & 15.0 & 0.62 & $0.62 \%$ & 45.0 & 235 & 15 & 75 & 0.11 & 1 & 1 & 0.083 & $0.07 \%$ & 4.3 & $\mathrm{RC}$ \\
\hline & VR13 & $\mathrm{S}$ & 7.5 & 15.0 & 0.62 & $0.62 \%$ & 45.0 & 235 & 15 & 75 & 0.11 & 1 & 1 & 0.083 & $0.07 \%$ & 4.4 & $\mathrm{RC}$ \\
\hline & VR14 & $\mathrm{S}$ & 7.5 & 15.0 & 0.62 & $0.62 \%$ & 45.0 & 235 & 15 & 75 & 0.11 & 1 & 1 & 0.083 & $0.07 \%$ & 3.9 & $\mathrm{RC}$ \\
\hline
\end{tabular}

\subsection{Design model in ULS}

The calculation methodology indicated in the ACI440.2R-17 [22] and FIB Model Code [23] standards was used to evaluate the bending safety factor of experimental data of reinforced concrete strengthened with CFRP.

Table 3 presents the criteria defined for such analysis, with 2 cases without partial safety factors (for comparison with the experimental data collected) and 2 cases with partial safety factors (verification of global safety). 
Table 3. Criteria defined for analysis.

\begin{tabular}{|c|c|c|c|c|}
\hline \multicolumn{5}{|c|}{ Parameters established for the analysis criteria } \\
\hline & \multicolumn{4}{|c|}{ Partial safety factor } \\
\hline & C.1 & C.2 & C.5 & C.6 \\
\hline Design equations. & $(\mathrm{ACI})$ & (FIB) & $(\mathrm{ACI})$ & (FIB) \\
\hline Material properties & $(\mathrm{ACI})$ & (FIB) & $(\mathrm{ACI})$ & (FIB) \\
\hline Ultimate compressive strain of concrete; $\left(\varepsilon_{\mathrm{cu}}\right)$ & $3 \%$ & $3.5 \%$ & $3 \%$ & $3.5 \%$ \\
\hline Limit of tensile strain in steel $\left(\varepsilon_{\mathrm{s}, \mathrm{lim}}\right)$ & - & - & - & - \\
\hline Strength reduction factor $(\phi)$ & 1 & 1 & $\left(\Delta \varepsilon_{\mathrm{s}}\right)$ & Verification \\
\hline FRP Strength reduction factor $(\psi f)$ & 1 & 1 & 0.85 & - \\
\hline Partial safety factor for steel $\left(\gamma_{\mathrm{s}}\right)$ & 1 & 1 & 1.10 & 1.15 \\
\hline Environmental reduction factor $\left(\mathrm{C}_{\mathrm{E}}\right)$ & 1 & 1 & 0.85 & 0.74 \\
\hline
\end{tabular}

As indicated in Figure 1, by imposing one of the failure modes and limiting the strain in the conditioning material, it is possible to determine the position of the neutral axis and obtain the effective strain in the FRP. Furthermore, the stresses and strains in the internal reinforcement in the FRP and in the concrete can be determined. However, in these calculations, it is necessary to consider the hypothesis of occurrence of premature failure characteristic in structures strengthened through EBR technique. Owing to the difficulty in detecting them, ACI440 [22] and FIB [23] limit the strain of FRP $\left(\varepsilon_{f d}\right)$ to increase the reliability of the reinforcement.

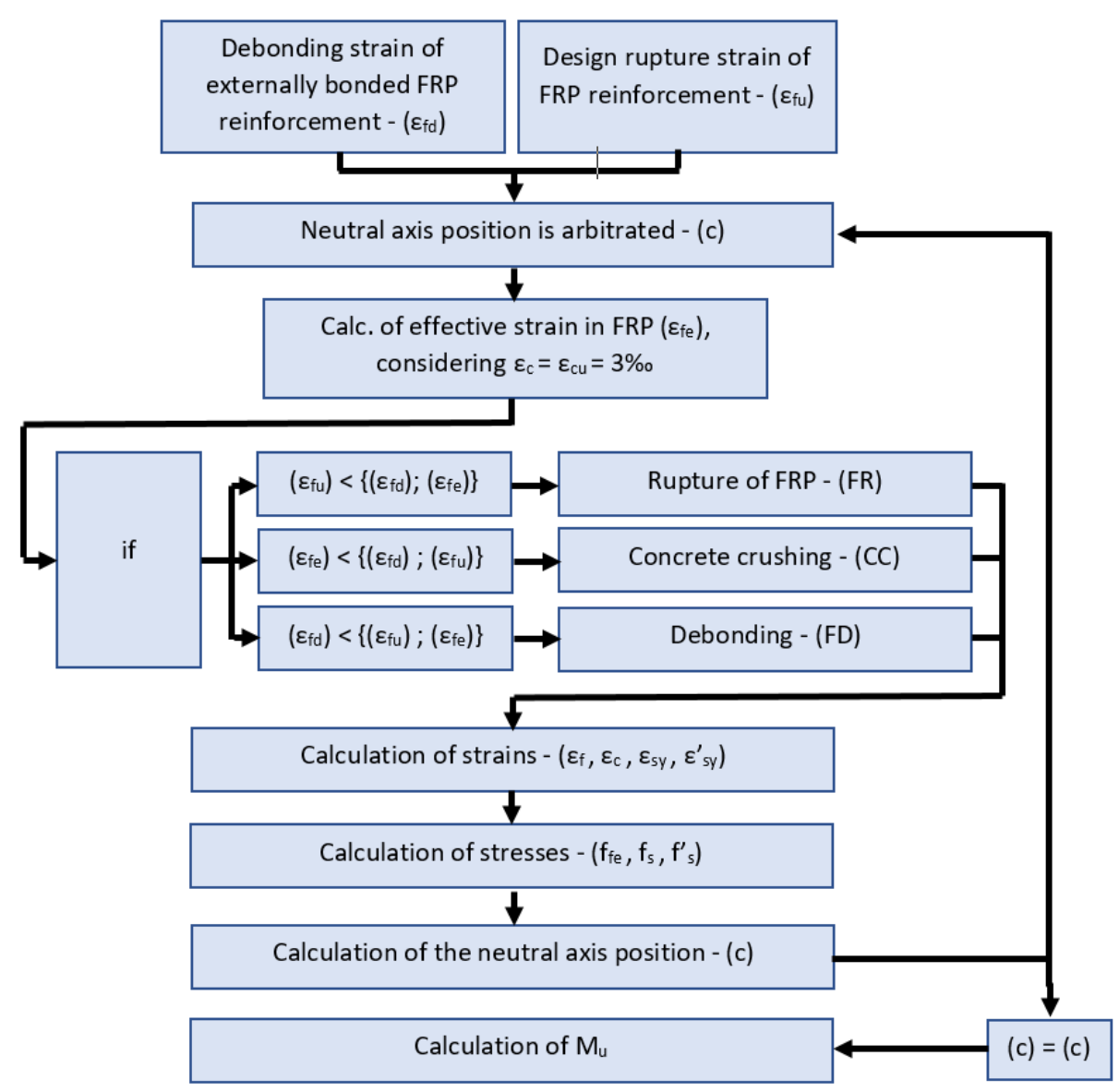

Figure 1. Flowchart of the calculation methodology adopted for the FIB and ACI models. 


\section{RESULTS AND DISCUSSIONS}

\subsection{Analysis of collected experimental data}

Note in Tables 1 and 2 that both for the slabs and for the beams, approximately $60 \%$ of the experimental data present premature rupture. The experimental data show that the slabs present larger strain values $\left(\varepsilon_{f u}\right)$ when compared to beams strengthened by the same externally bonded technique (EBR).

In the slabs case, given that the shear is not very pronounced in these models, the strain in CFRP shows high values ( $\varepsilon_{\text {med }} \approx 11 \%$ ), especially when rupture is controlled by classical failure. However, even in situations of premature failure, accurate results can be observed from these experimental data $\left(\varepsilon_{\text {med }} \approx 9 \%\right.$ ).

These results are evidenced in studies of several authors, namely ACI-440 [22], Juvandes [24], FIB-14 [35], Brosens [39], Matthys [40], and Azevedo [43], concluding that in beam models the develops lower strain values than in the slab models.

Important to note that the collection of data presented in this paper reveals an interesting variation in reported test results. As presented by Naser et al. [15], this can be attributed to significant variation in tested specimens, material types, loading configurations, experimental procedures, and test arrangements, etc., which make interpretation of test results complex. This demanded a standardization on testing procedures, and some tests are published, [44] and [45].

\subsection{Ultimate flexural strength analysis (ACI x FIB)}

To perform the statistical analysis of the ratio between the ultimate theoretical and experimental moments, the proportion ( $M_{\text {theoretical }} / M_{\text {exp. }}$ ) of the 64 experimental data was assessed in this study for the corresponding criteria (C.1 and C.2). Such values are plotted in Figure 2, 3 and 4. The mean, standard deviation (SD), and coefficient of variation (CV) were calculated; they are presented in Table 4.

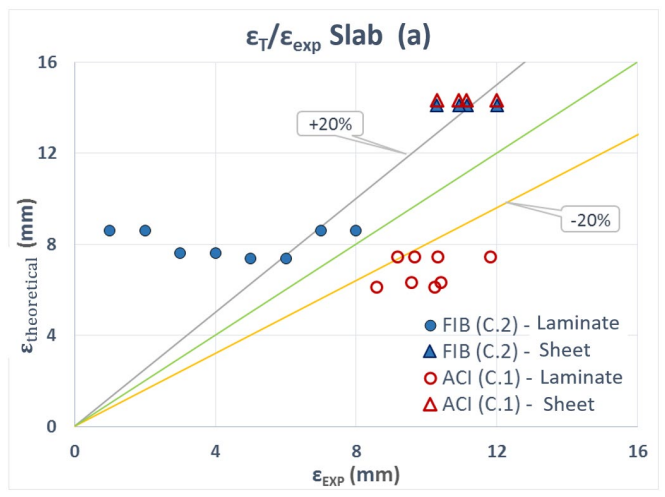

Figure 2. ACI440 x FIB - Analysis of the ultimate strain ( $\left.\epsilon_{\text {theoretical }} / \epsilon_{\text {exp }}\right)$ for slabs (a).

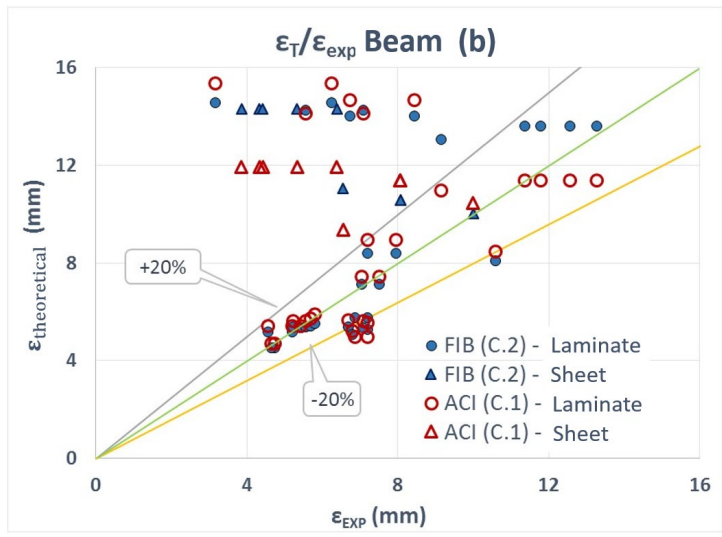

Figure 3. ACI440 x FIB - Analysis of the ultimate strain $\left(\epsilon_{\text {theoretical }} / \epsilon_{\text {exp }}\right)$ for beam (b). 


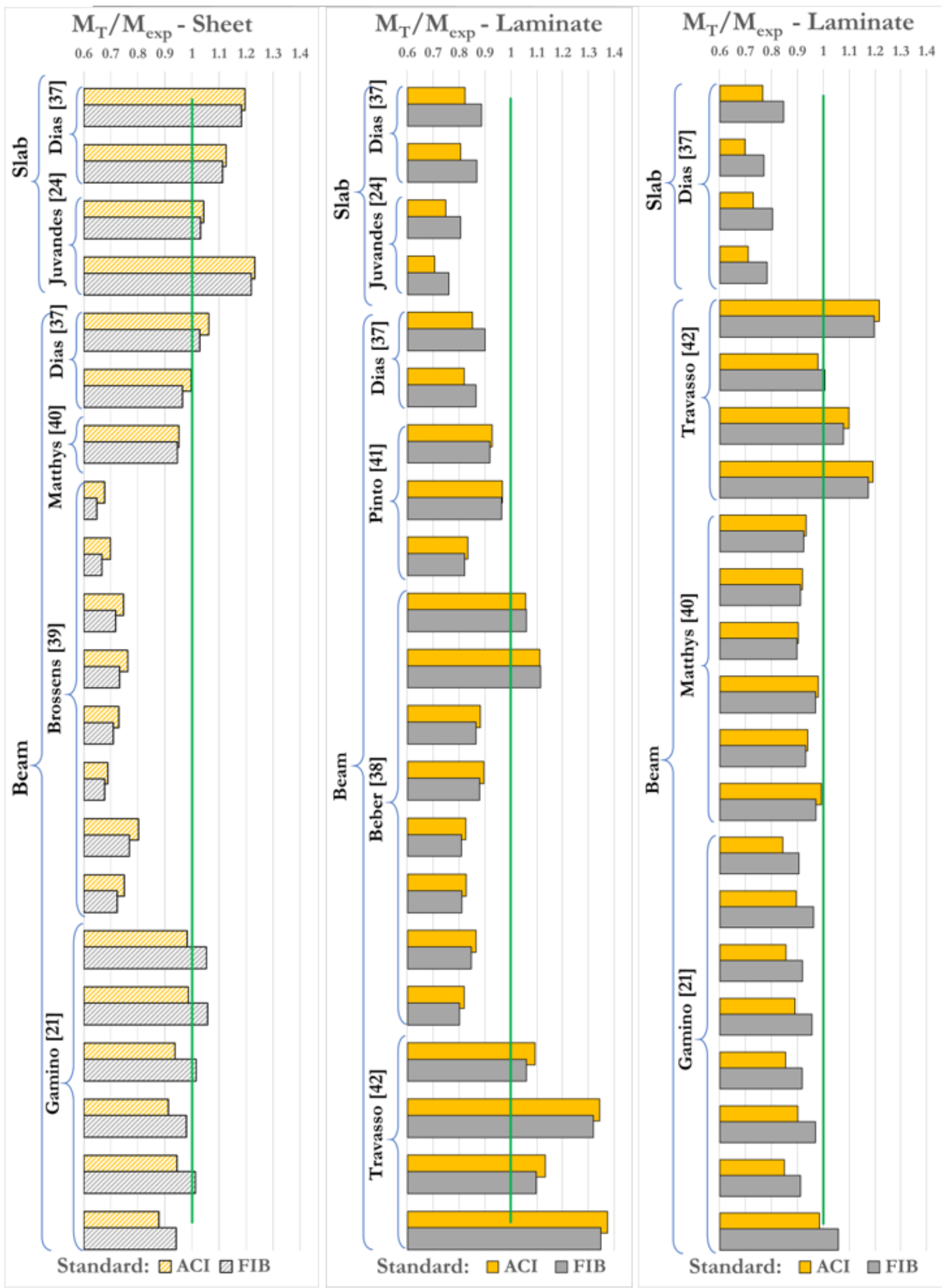

Figure 4. ACI440 x FIB - Analysis of the ultimate moment of the 64 experimental data $\left(M_{\text {theoretical }} / M_{\text {exp }}\right)$. 
Table 4. Mean, standard deviation (SD), and coefficient of variation $(\mathrm{CV})$ of the ratios $\left(M_{\text {theoretical }} / M_{\text {exp }}\right)$ and $\left(\epsilon_{\text {theoretical }} / \epsilon_{\text {exp }}\right)$ FIB x ACI.

\begin{tabular}{|c|c|c|c|c|c|c|c|c|c|c|c|}
\hline \multicolumn{12}{|c|}{$\left(M_{\text {theoret. }} / M_{\text {exp }}\right)$ of 64 experimental data } \\
\hline & & & & & & & & & & ACI & FIB \\
\hline & & & & & & & & & & (C.1) & (C.2) \\
\hline & & & & \multirow{2}{*}{\multicolumn{3}{|c|}{$\begin{array}{r}\text { ACI } \\
\text { (C.1) }\end{array}$}} & FIB & \multirow{3}{*}{$\begin{array}{c}\text { Slab } \\
\text { (Laminates) }\end{array}$} & Mean & 0.75 & 0.82 \\
\hline & & & & & & & (C.2) & & SD & 0.05 & 0.05 \\
\hline & & & & \multirow{3}{*}{$\begin{array}{l}\text { Total } \\
\text { Slab }\end{array}$} & Mean & 0.88 & 0.92 & & $\mathrm{CV}(\%)$ & 6.28 & 5.71 \\
\hline & & ACI & FIB & & SD & 0.21 & 0.17 & \multirow{3}{*}{ Slab (Sheet) } & Mean & 1.15 & 1.14 \\
\hline & & (C.1) & (C.2) & & $\mathrm{CV}(\%)$ & 23.31 & 18.19 & & $\mathrm{SD}$ & 0.08 & 0.08 \\
\hline \multirow{7}{*}{ Total } & Mean & 0.92 & 0.95 & \multirow{4}{*}{$\begin{array}{l}\text { Total } \\
\text { Beam }\end{array}$} & & & & & $\mathrm{CV}(\%)$ & 7.23 & 7.23 \\
\hline & SD & 0.15 & 0.15 & & Mean & 0.93 & 0.96 & \multirow{3}{*}{$\begin{array}{c}\text { Beam } \\
\text { (Laminates) }\end{array}$} & Mean & 0.97 & 0.98 \\
\hline & $\mathrm{CV}(\%)$ & 16.73 & 16.04 & & $\mathrm{SD}$ & 0.15 & 0.15 & & SD & 0.15 & 0.13 \\
\hline & & & & & $\mathrm{CV}(\%)$ & 16.02 & 15.71 & & $\mathrm{CV}(\%)$ & 15.11 & 13.73 \\
\hline & & & & & & & & \multirow{3}{*}{ Beam (Sheet) } & Mean & 0.85 & 0.86 \\
\hline & & & & & & & & & SD & 0.13 & 0.16 \\
\hline & & & & & & & & & $\mathrm{CV}(\%)$ & 14.88 & 18.20 \\
\hline \multicolumn{12}{|c|}{ ( $\varepsilon_{\text {theoret. }} / \varepsilon_{\text {exp } . ~}$ ) of 52 experimental data* } \\
\hline & & & & & & & & & & ACI & FIB \\
\hline & & & & & & & & & & (C.1) & (C.2) \\
\hline & & & & \multirow{2}{*}{\multicolumn{3}{|c|}{$\begin{array}{c}\text { ACI } \\
\text { (C.1) }\end{array}$}} & FIB & \multirow{3}{*}{$\begin{array}{c}\text { Slab } \\
\text { (Laminates) }\end{array}$} & Mean & 0.69 & 0.81 \\
\hline & & & & & & & (C.2) & & SD & 0.08 & 0.08 \\
\hline & & & & \multirow{3}{*}{$\begin{array}{l}\text { Total } \\
\text { Slab }\end{array}$} & Mean & 0.89 & 0.97 & & $\mathrm{CV}(\%)$ & 11.39 & 10.17 \\
\hline & & ACI & FIB & & $\mathrm{SD}$ & 0.31 & 0.24 & \multirow{3}{*}{ Slab (Sheet) } & Mean & 1.30 & 1.27 \\
\hline & & (C.1) & (C.2) & & $\mathrm{CV}(\%)$ & 34.65 & 24.89 & & SD & 0.08 & 0.08 \\
\hline \multirow{7}{*}{ Total } & Mean & 1.32 & 1.38 & \multirow{4}{*}{$\begin{array}{l}\text { Total } \\
\text { Beam }\end{array}$} & & & & & $\mathrm{CV}(\%)$ & 6.27 & 6.27 \\
\hline & SD & 0.79 & 0.84 & & Mean & 1.44 & 1.50 & \multirow{3}{*}{$\begin{array}{c}\text { Beam } \\
\text { (Laminates) }\end{array}$} & Mean & 1.29 & 1.28 \\
\hline & $\mathrm{CV}(\%)$ & 60.19 & 61.08 & & SD & 0.85 & 0.92 & & SD & 0.83 & 0.78 \\
\hline & & & & & $\mathrm{CV}(\%)$ & 58.82 & 61.09 & & $\mathrm{CV}(\%)$ & 64.21 & 60.62 \\
\hline & & & & & & & & \multirow{3}{*}{ Beam (Sheet) } & Mean & 1.99 & 2.27 \\
\hline & & & & & & & & & SD & 0.73 & 1.00 \\
\hline & & & & & & & & & CV $(\%)$ & 36.53 & 43.99 \\
\hline
\end{tabular}

* 12 of 64 experimental strain data collected $\left(\varepsilon_{\exp }\right)$ are unknown.

According to Figure 4 and Table 4, concerning the cases of slabs, it is observed that the FIB model code [23] yields more consistent results (ratio near to 1.0) than the ACI440 model [22]. In the case of slabs strengthened with laminate, the prediction of the moment capacity of the slab is quite conservative according to these two philosophies, and the ACI440 [22] proved to be more conservative than FIB [23]. Note that while the ACI [22] criterion is generally more conservative than FIB [23], both seem to better reflect the behavior of beams than the behavior of slabs (expressed by a ratio $M_{\text {theoretical }} / M_{\text {exp }}<1,00$, and by values closer to the experimental results and with less dispersion). This can be explained by the fact that in slabs the shear is not pronounced, making the FRP more effective in these cases. Since two guidelines do not differentiate between beams and slabs; the estimated failure moment values are over conservative for the slabs case. Itis also noted a difference in the behavior between sheets and laminates, demonstrating the need to separate their specification in a future code.

Concerning beams strengthened with laminates, the ultimate behavior of design models is foreseen with high accuracy ( $M_{\text {theoretical }} / M_{\text {exp }} \approx 1.0$ ) and low dispersion $(\mathrm{CV} \approx 15 \%$ ) according to the ACI and FIB criteria. In contrast, the models reinforced with sheets lead to more conservative ultimate moments average.

As observed in Table 4, the increase on the concrete ultimate strain from $3.0 \%$ (ACI [22]) to $3.5 \%$ (FIB [23]), set out in criterion C.2, resulted in a small increase in the mean ratio $\left(M_{\text {theoret. }} / M_{\text {exp }}\right)$ of the 64 elements, due considering the remaining resistance in the compression of the reinforced element. This demonstrates the importance of assessing the strength reserve in the compression of a structure to be strengthened, being, in most cases, the mandatory factor of design to strength a structure. To be noted that ABNT NBR6118 [36] specifies the same concrete ultimate strain value as FIB [23]. 
In general, when compared to the ACI440.2R-17 model, the FIB model code [23] presents a smaller dispersion of values and analytical results closer to the experimental values, expressed by the ratio $M_{\text {theoretical }} / M_{\text {exp }}$ near 1 presented on Table 4. Some of these evidences agrees with the studies by Pham and Al-Mahaidi [46] and Toutanji et al. [47].

\subsection{Analysis of maximum strain of FRP (ACI x FIB)}

From Figure 2, Figure 3, and Table 4, comparing beams and slabs, it can be concluded that in both codes the slabs presented ultimate strain with a closer and conservative approximation of the theorical results when compared to experimental results, and with a reasonable dispersion of values, reflected by an average value of 0.89 (ACI) / 0.97 (FIB) and a CV of $34.65 \%$ (ACI) / 24.89\% (FIB). The beams analyses led to theoretical values higher than those obtained in the experimental tests. This difference in behavior between beams and slabs can be explained because of the occurrence of premature failures earlier than expected in the beam models.

When compared to the FRP sheet models, the FRP laminates showed a better approximation on the ultimate theorical strain compared with experimental results (for beams and slabs).

Comparing the ACI and FIB models, it can be concluded that for slabs the FIB model fits better to the maximum strain of the FRP, with an average of the ratio ( $\left.\varepsilon_{\text {theoretical }} / \varepsilon_{\text {exp }}\right)$ closer to 1 and a smaller dispersion of results than the ACI model. However, in general, as observed in Figure 2 and 3, different from the relation between the ultimate moments ( $\left.M_{\text {theoretical }} / M_{\text {exp }}\right)$, the strain shows remarkable dispersion of values $(\mathrm{CV}>50 \%)$, proving not to be a good parameter for analysis and convergence of values.

\subsection{Analysis of the global safety factor (ACI x FIB)}

To carry out the analysis of the global safety factor, it was calculated the ratio C.1/C.5 for ACI and C.2/C.6 for FIB, where C. 1 and C. 2 are the ultimate moment of the strengthened member without consideration of partial safety factor and C.5 and C. 6 are the same moment including partial safety factors, as shown in Table 5.

Table 5. Global safety factor obtained - FIB x ACI.

\begin{tabular}{|c|c|c|c|c|c|c|c|c|}
\hline \multicolumn{9}{|c|}{ Global safety factor (C.1/C.5) - ACI } \\
\hline & & & & & & \multirow{3}{*}{ FR } & Mean & 1.35 \\
\hline & & & \multirow{3}{*}{ Slab } & Mean & 1.31 & & $\mathrm{SD}$ & 0.06 \\
\hline & & & & SD & 0.07 & & $\mathrm{CV}(\%)$ & 4.19 \\
\hline & Mean & 1.28 & & $\mathrm{CV}(\%)$ & 5.66 & \multirow{3}{*}{ FD } & Mean & 1.27 \\
\hline \multirow[t]{10}{*}{ Total } & $\mathrm{SD}$ & 0.05 & & & & & $\mathrm{SD}$ & 0.04 \\
\hline & $\mathrm{CV}(\%)$ & 3.97 & \multirow{3}{*}{ Beam } & Mean & 1.27 & & $\mathrm{CV}(\%)$ & 3.20 \\
\hline & & & & $\mathrm{SD}$ & 0.04 & \multirow{3}{*}{$\mathrm{CC}$} & Mean & 1.25 \\
\hline & & & & $\mathrm{CV}(\%)$ & 3.12 & & SD & 0.02 \\
\hline & & & & & & & $\mathrm{CV}(\%)$ & 1.24 \\
\hline & & & \multirow{2}{*}{\multicolumn{3}{|c|}{ Global safety factor (C.2/C.6) - FIB }} & & & \\
\hline & & & & & & \multirow{3}{*}{ FR } & Mean & 1.35 \\
\hline & & & \multirow{3}{*}{ Slab } & Mean & 1.26 & & SD & 0.03 \\
\hline & & & & SD & 0.09 & & $\mathrm{CV}(\%)$ & 2.40 \\
\hline & Mean & 1.32 & & $\mathrm{CV}(\%)$ & 7.25 & \multirow{3}{*}{ FD } & Mean & 1.24 \\
\hline \multirow[t]{5}{*}{ Total } & SD & 0.08 & & & & & $\mathrm{SD}$ & 0.06 \\
\hline & $\mathrm{CV}(\%)$ & 5.88 & \multirow{3}{*}{ Beam } & Mean & 1.33 & & $\mathrm{CV}(\%)$ & 5.01 \\
\hline & & & & SD & 0.07 & \multirow{3}{*}{$\mathrm{CC}$} & Mean & 1.37 \\
\hline & & & & $\mathrm{CV}(\%)$ & 5.40 & & SD & 0.03 \\
\hline & & & & & & & $\mathrm{CV}(\%)$ & 2.47 \\
\hline
\end{tabular}


Table 5 shows an average value of 1.28 for the global safety factor obtained for the ACI (C.1/C.5). However, when comparing the global safety factors of ACI440, and separating them by the type of failure, note that the largest global safety factor occurs when the analytical mode failure is due to the rupture of the FRP (FR). This can be explained by the environmental reduction factor (CE) being directly associated with this mode of failure. Concrete crushing presented the lowest global safety factor, but still with values within the parameters found in the literature.

From Table 5, the global safety factor obtained (C.2/C.6) for the FIB Model Code [22] is a mean value of 1.32. However, the highest global safety factor occurs when the failure mode of the structure is due to the rupture of the FRP and concrete crushing. This can be explained due to the partial safety factor of the FRP material $\left(y_{f}\right)$, which limits the maximum strain of the FRP, being directly associated with this mode of failure (FR). For concrete crushing, the partial safety factor of concrete $y_{c}$ is directly associated with the global safety factor values, and according to FIB-14 [35], this value is set as $y_{c}=1.5$.

\section{CONCLUSIONS}

- The design procedures presented in the ACI440 and FIB Model Code design guides have differences in the approach of the FRP subject, however, without large discrepancies of results, considering the FRP to be a recent reinforcement system. The importance of this topic demonstrates the need to create codes on these matters in Brazil.

- The FRP strain analysis ( $\varepsilon_{\text {theoretical }} / \varepsilon_{\text {exp }}$ ) presented large coefficient of variation, proving not to be a good parameter to be assessed for convergence of values. Therefore, future regulation in Brazil should be mainly based on flexural strength principles.

- For structures strengthened with FRP by the EBR technique, when compared to ACI440 design guide, the design methodology specified by FIB Model Code leads to analytical results closer to the experimental values, with smaller results dispersion, expressed as the ratio $M_{\text {theoret. }} / M_{\text {exp }}$ close to 1.0 .

- Both design models (ACI440 and FIB) seem to better reflect the behavior of beams than slabs. The account for the different element types (slabs/beams and laminates/sheets) is not considered in the FIB neither ACI models nor should be developed in future studies in Brazil.

- It is noted that in 9 of 64 experimental data that the rupture of the member started after excessive steel yielding, and in one of these cases the failure was due only to excessive plastic deformation of steel, pointing to a need to limit the excessive steel yielding, especially in cases where the amount of reinforcement is an important factor for the service life of the structure. ABNT NBR6118 limits the strain of the reinforcement to ( $\varepsilon_{s y, l i m}=10 \%$ ), and this may be an important factor to be adopted in a future Brazilian codes on FRP;

- In general, the Brazilian code for Concrete Structures is like the European standard (Eurocode) one. Therefore, the FIB Model Code 2010 model presents a calculation methodology and partial safety factors that can be more easily related to the future Brazilian code on Reinforced Concrete Structures Strengthened With CFRP.

\section{ACKNOWLEDGEMENTS}

The authors are grateful for the support of Brazilian Research Funding Agencies - CNPq (Conselho Nacional de Desenvolvimento Científico e Tecnológico) - scholarship number 152486/2016-0.

\section{REFERENCES}

[1] A. Siddika, M. A. A. Mamun, W. Ferdous, and R. Alyousef, "Performances, challenges and opportunities in strengthening reinforced concrete structures by using FRPs: a state-of-the-art review," J. Eng. Fail. Anal., vol. 111, pp. 104480, 2020, http://dx.doi.org/10.1016/j.engfailanal.2020.104480.

[2] H. Toutanji, L. Zhao, and E. Anselm, "Verifications of design equations of beams externally strengthened with FRP composites," $J$. Compos. Constr., vol. 10, no. 3, pp. 254-264, 2006, http://dx.doi.org/10.1061/(ASCE)1090-0268(2006)10:3(254).

[3] U. Meier and H. Kaiser, "Strengthening of structures with CFRP laminates," in Proc. Adv. Compos. Mater. Civ. Eng. Struct., New York, 1991, pp. 224-232.

[4] P. Ritchie, D. Thomas, L. Lu, and G. Conneley, "External reinforcement of concrete beams using fiber reinforced plastics," $A C I$ Struct. J., vol. 88, no. 4, pp. 490-500, 1991, http://dx.doi.org/10.14359/2723.

[5] A. Sharif, G. Al-Sulaimani, I. Basunbul, M. Bakuch, and B. Ghaleb, "Strengthening of initially loaded reinforced concrete beams using FRP plates," ACI Struct. J., vol. 91, no. 2, pp. 160-168, 1994, http://dx.doi.org/10.14359/4594. 
[6] A. Siddika, M. A. A. Mamun, R. Alyousef, and Y. H. M. Amran, "Strengthening of reinforced concrete beams by using fiberreinforced polymer composites: a review," J. Build. Eng., vol. 25, pp. 100798, 2019, http://dx.doi.org/10.1016/j.jobe.2019.100798.

[7] L. Huang, C. Zhang, L. Yan, and B. Kasal, "Flexural behavior of U-shape FRP profile-RC composite beams with inner GFRP tube confinement at concrete compression zone," Compos. Struct., vol. 184, pp. 674-687, 2018, http://dx.doi.org/10.1016/j.compstruct.2017.10.029.

[8] U. Meier, "Carbon fiber-reinforced polymers: modern materials in bridge engineering," Struct. Eng. Int., vol. 2, no. 1, pp. 7-12, 1992, http://dx.doi.org/10.2749/101686692780617020.

[9] L. Sorrentino, S. Turchetta, and C. Bellini, "In process monitoring of cutting temperature during the drilling of FRP laminate," Compos. Struct., vol. 168, pp. 549-561, 2017, http://dx.doi.org/10.1016/j.compstruct.2017.02.079.

[10] J. Huo, Z. Li, L. Zhao, J. Liu, and Y. Xiao, "Dynamic behavior of CFRP-strengthened reinforced concrete beams without stirrups under impact loading," ACI Struct. J., vol. 115, no. 3, pp. 775-787, 2018, http://dx.doi.org/10.14359/51701283.

[11] Y. Ou and D. Zhu, "Tensile behavior of glass fiber reinforced composite at different strain rates and temperatures," Constr. Build. Mater., vol. 96, pp. 648-656, 2015., http://dx.doi.org/10.1016/j.conbuildmat.2015.08.044.

[12] D. A. Bournas, A. Pavese, and W. Tizani, "Tensile capacity of FRP anchors in connecting FRP and TRM sheets to concrete," Eng. Struct., vol. 82, pp. 72-81, 2015, http://dx.doi.org/10.1016/j.engstruct.2014.10.031.

[13] B. H. Osman, E. Wu, B. Ji, and S. S. Abdulhameed, "Repair of pre-cracked reinforced concrete (RC) Beams with openings strengthened using FRP sheets under sustained load," Int. J. Concr. Struct. Mater., vol. 11, no. 1, pp. 171-183, 2017., http://dx.doi.org/10.1007/s40069-016-0182-3.

[14] Y. H. Mugahed Amran, R. Alyousef, R. S. M. Rashid, H. Alabduljabbar, and C.-C. Hung, "Properties and applications of FRP in strengthening RC structures: a review," Structures, vol. 16, pp. 208-238, 2018, http://dx.doi.org/10.1016/j.istruc.2018.09.008.

[15] M. Z. Naser, R. A. Hawileh, and J. A. Abdalla, "Fiber-reinforced polymer composites in strengthening reinforced concrete structures: a critical review," J. Eng. Struct., vol. 198, pp. 109542, 2019, http://dx.doi.org/10.1016/j.engstruct.2019.109542.

[16] L. Czarnecki, M. Kaproń, and D. Van Gemert, "Sustainable construction: challenges, contribution of polymers, research arena," Restor. Build Monuments, vol. 19, no. 2-3, pp. 81-96, 2013, http://dx.doi.org/10.1515/rbm-2013-6583.

[17] M. F. Humphreys, "The use of polymer composites in construction," in Int. Conf. Smart \& Sustain. Built Environ., Brisbane, Australia, 2003.

[18] R. A. Hawileh, W. Nawaz, and J. A. Abdalla, "Flexural behavior of reinforced concrete beams externally strengthened with Hardwire Steel-Fiber sheets," Constr. Build. Mater., vol. 172, pp. 562-573, 2018, http://dx.doi.org/10.1016/j.conbuildmat.2018.03.225.

[19] R. A. Hawileh, J. A. Abdalla, and M. Z. Naser, "Modeling the shear strength of concrete beams reinforced with CFRP bars under unsymmetrical loading," Mech. Adv. Mater. Structures, vol. 26, no. 1, pp. 1-8, 2018.

[20] H. Rasheed, J. A. Abdalla, R. Hawileh, and A. Al-Tamimi, "Flexural behavior of reinforced concrete beams strengthened with externally bonded aluminum alloy plates," Eng. Struct., vol. 147, no. 15, pp. 473-485, 2017, http://dx.doi.org/10.1016/j.engstruct.2017.05.067.

[21] A. L. Gamino, T. N. Bittencourt, and J. L. A. O. Sousa, "Estruturas de concreto reforçadas com PRFC. Parte I: análise dos modelos de flexão," Rev. IBRACON, vol. 2, no. 4, pp. 326-355, 2009, http://dx.doi.org/10.1590/S1983-41952009000400003.

[22] American Concrete Institute, Guide for the Design and Construction of Externally Bonded FRP Systems for Strengthening Concrete Structure, ACI 440.2R-17, 2017.

[23] Federation International Du Béton, FIB Model Code for Concrete Structures 2010, 2010.

[24] L. F. P. Juvandes, "Reforço e reabilitação de estruturas de betão usando materiais compósitos de CFRP,” Ph.D. dissertation, Fac. Eng., Univ. Porto, 1999.

[25] H. A. Rasheed, Strengthening Design of Reinforced Concrete with FRP. Boca Raton: CRC Press, 2014.

[26] L. C. Hollaway, "A review of the present and future utilization of FRP composites in the Civil infrastructure with reference to their important in service properties," Constr. Build. Mater., vol. 24, no. 12, pp. 2419-2445, 2010, http://dx.doi.org/10.1016/j.conbuildmat.2010.04.062.

[27] L. De Lorenzis and J. G. Teng, "Near-surface mounted FRP reinforcement: an emerging technique for strengthening structures," Compos. B. Eng., vol. 38, no. 2, pp. 119-143, 2007., http://dx.doi.org/10.1016/j.compositesb.2006.08.003.

[28] R. Kalfat, R. Al-Mahaidi, and S. T. Smith, "Anchorage devices used to improve the performance of reinforced concrete beams retrofitted with FRP composites: stateof- the-art review," J. Compos. Constr., vol. 17, no. 1, pp. 14-33, 2013. http://dx.doi.org/10.1061/(ASCE)CC.1943-5614.0000276.

[29] H. Pham and R. Al-Mahaidi, "Experimental investigation into flexural retrofitting of reinforced concrete bridge beams using FRP composites," Compos. Struct., vol. 66, no. 1-4, pp. 617-625, 2004., http://dx.doi.org/10.1016/j.compstruct.2004.05.010.

[30] E. del Rey Castillo, M. Griffith, and J. Ingham, "Straight FRP anchors exhibiting fiber rupture failure mode," Compos. Struct., vol. 207, pp. 612-624, 2019. 
[31] A. M. Okeil, Y. Bingol, and T. Alkhrdahi, Analyzing Model Uncertainties for Concrete Beams Flexurally Strengthened with FRP Laminates. Washington, DC., 2007.

[32] M. Arduini and A. Nanni, "Parametric study of beams with externally bonded FRP reinforcement," ACI Struct. J., vol. 94, no. 5, pp. 493-501, 1997, http://dx.doi.org/10.14359/499.

[33] H. Toutanji, L. Zhao, and Y. Zhang, "Flexural behavior of reinforced concrete beams externally strengthened with CFRP sheets bonded with an inorganic matrix," Eng. Struct., vol. 28, no. 4, pp. 557-566, 2006, http://dx.doi.org/10.1016/j.engstruct.2005.09.011.

[34] A. K. Al-Tamimi, R. Hawileh, J. Abdalla, and H. A. Rasheed, "Effects of ratio of CFRP plate length to shear span and end anchorage on flexural behavior of SCC RC beams," J. Compos. Constr., vol. 15, no. 6, pp. 908-919, 2011, http://dx.doi.org/10.1061/(ASCE)CC.1943-5614.0000221.

[35] Federation International Du Béton, Bulletin 14: Externally bonded FRP reinforcement for RC structures, 2001.

[36] Associação Brasileira de Normas Técnicas, Projeto de Estruturas de Concreto, NBR 6118, 2014.

[37] S. Dias, "Verificação experimental do reforço com CFRP de estruturas de betão à flexão," M.S. thesis, Fac. Eng., Univ. Porto (FEUP), Porto, 2001.

[38] A. Beber, “Avaliação do Desempenho de Vigas de Concreto Armado Reforçadas com Lâminas de Fibra de Carbono," Ph.D. dissertation, Univ. Fed. Rio Grande do Sul (UFRGS), Porto Alegre, 1999.

[39] K. Brosens, “Anchorage of externally bonded steel plates and CFRP laminates for the strengthening of concrete elements," Ph.D. dissertation, Katholieke Univ. Leuven, Leuven, Bélgica, 2001.

[40] S. Matthys, "Structural behaviour and design of concrete members strengthened with externally bonded FRP reinforcement," Ph.D. dissertation, Ghent Univ., Ghent, 2000.

[41] C. Pinto, "Reforço à flexão de vigas de concreto armado com fibras de carbono," M.S. thesis, Univ. Fed. Rio de Janeiro, Rio de Janeiro, 2000.

[42] N. Travassos, “Caracterização do comportamento da ligação CFRP-Betão,” M.S. thesis, Inst. Superior Técnico (IST), Lisboa, 2005.

[43] D. M. M. Azevedo, "Reforço de estruturas de betão com colagem de sistemas compósitos de CFRP - Recomendações para dimensionamento," M.S. thesis, Fac. Eng., Univ. Porto (FEUP), Porto, 2008.

[44] American Society for Testing and Materials, Standard Guide for Testing Polymer Matrix Composite Materials, ASTM D4762-16, 2016.

[45] International Organization for Standardization, Fibre-Reinforced Plastics - Methods of Producing Test Plates - Part 1: General Conditions, ISO 1268-1:2001, 2001.

[46] H. Pham and R. Al-Mahaidi, "Assessment of available prediction models for the stength of FRP retrofitted RC beams," Compos. Struct. J., vol. 66, no. 1-4, pp. 601-610, 2004.

[47] H. Toutanji and L. Zhao, "Review of design equations of beams externally strengthened with FRP composites," in Intl. Wksh. Innov. Mater. Des., Cairo, Egypt, 2005. https://doi.org/10.1061/(ASCE)1090-0268(2006)10:3(254).

Author contributions: IDGO: conceptualization, analysis, methodology, data curation, writing, TNB and LCM: conceptualization, analysis, methodology, reviewer, supervision;

Editors: Mark Alexander, Guilherme Aris Parsekian. 